\begin{tabular}{|c|l|}
\hline Title & Twin formation in hematite during dehy dration of goethite \\
\hline Author(s) & Saito, Genki; Kunisada, Yuji; Nomura, Takahiro; Sakaguchi, Norihito; A kiyama, Tomohiro \\
\hline Citation & $\begin{array}{l}\text { Physics and chemistry of minerals, 43(10), 749-757 } \\
\text { https://doi.org/10.1007/300269-016-0831-8 }\end{array}$ \\
\hline Issue Date & 2016-11 \\
\hline Doc URL & http://hdl.handle.net/2115/67493 \\
\hline Rights & The final publication is available at link.springer.com \\
\hline Type & article (author version) \\
\hline File Information & manuscript.pdf \\
\hline
\end{tabular}

Instructions for use 


\section{Twin formation in hematite during dehydration of goethite}

Genki Saito ${ }^{*}$, Yuji kunisada, Takahiro Nomura, Norihito Sakaguchi, Tomohiro Akiyama

${ }^{a}$ Center for Advanced Research of Energy and Materials, Hokkaido University, Sapporo 060-8628, Japan

* Corresponding Author: E-mail: genki@eng.hokudai.ac.jp Tel.: +81-11-706-6766 


\begin{abstract}
Twin formation in hematite during dehydration was investigated using X-ray diffraction, electron diffraction, and high-resolution transmission electron microscopy (TEM). When synthetic goethite was heated at different temperatures between 100 and $800{ }^{\circ} \mathrm{C}$, a phase transformation occurred at temperatures above $250^{\circ} \mathrm{C}$. The electron diffraction patterns showed that the single-crystalline goethite with a growth direction of $[001]_{\mathrm{G}}$ was transformed into hematite with a growth direction of $[100]_{\mathrm{H}}$. Two non-equivalent structures emerged in hematite after dehydration, with twin boundaries at the interface between the two variants. As the temperature was increased, crystal growth occurred. At $800{ }^{\circ} \mathrm{C}$, the majority of the twin boundaries disappeared; however, some hematite particles remained in the twinned variant. The electron diffraction patterns and high-resolution TEM observations indicated that the twin boundaries consisted of crystallographically equivalent prismatic (100), (010), and (1̄̄0) planes. According to the total energy calculations based on spin-polarized density functional theory, the twin boundary of prismatic (100) screw had small interfacial energy $\left(0.24 \mathrm{~J} / \mathrm{m}^{2}\right)$. Owing to this low interfacial energy, the prismatic (100) screw interface remained after higher-temperature treatment at $800{ }^{\circ} \mathrm{C}$.
\end{abstract}

Keywords: dehydration, twinning, transformation, goethite, hematite, thermal decomposition, twin boundary 


\section{Introduction}

Goethite $(\alpha \mathrm{FeOOH})$ is widely distributed in soils, rocks, sediments, and ores [1, 2], and it can be a source material for catalysts [3], magnetic materials [4], and in iron production [5]. In the past few decades, the thermal decomposition of goethite into hematite has been reported from various viewpoints, including kinetics analysis [6], spectroscopy [7, 8], diffractometry [9, 10], and electron microscopy [11]. Studies using electron microscopy and X-ray diffraction (XRD) have further clarified the transformation from goethite to hematite [6, 11]. Microscopic observations have also clarified the thermal decomposition of goethite into hematite through the formation mechanism of slit-like micropores [12]. In this case, the slits are generated along the goethite $[001]_{G}$ direction, where the crystal is defined by the orthorhombic space group Pbnm, with lattice constants of $a_{\mathrm{G}}=4.5979 \AA, b_{\mathrm{G}}=9.9510 \AA$, and $c_{\mathrm{G}}=3.0175 \AA$ [12-14]. Structural investigation on the thermal decomposition of goethite was carried out using X-ray and electron diffraction with high-resolution transmission electron microscope (TEM) imaging. The topotactic relationship between goethite and hematite was revealed to be $(100)_{\mathrm{G}} / /(001)_{\mathrm{H}}$ by van Oosterhout [15]. Furthermore, Francombe and Rooksby clarified the unit cell relationships between goethite and hematite, as did Gualtieri and Venturelli in a later study $[10,16]$. When goethite is thermally dehydrated at lower temperatures, the mosaic polycrystalline structure consists of aggregates of well-oriented, twin-related hematite crystals, in which the twin-related hematite crystals are separated by $(001)_{\mathrm{H}}$ walls owing to the loss of water. Moreover, the twin boundary was found to be $(100)_{\mathrm{H}}$, as demonstrated by high-resolution electron microscopy [11, 17]. In this study, twin formation in hematite during dehydration was investigated, in which goethite samples were dehydrated at different temperatures between 100 and $800{ }^{\circ} \mathrm{C}$. Room temperature XRD was carried out to study the phase and crystal size. To investigate the detailed crystal structure including twin boundary, this study employed high resolution TEM and electron diffraction analysis. In situ dehydration of goethite together with TEM was also carried out to confirm the topotactic relationship between 
goethite and hematite, because XRD only analyses the agglomerated particles. When the electron beam is strongly focused on the goethite particles during TEM, the particles are partially heated and then dehydrated. By analyzing the high resolution TEM image and electron diffraction pattern, we can investigate the topotactic relationship during dehydration. Finally, the interfacial energy of the obtained twin boundary was analyzed by performing total energy calculations based on spin-polarized density functional theory (DFT).

\section{Experiment}

Reagent goethite powder, with a purity of $99 \%$ and a particle size of $1 \mu \mathrm{m}$, was obtained from the Kojundo Chemical Lab. Co. and used to prepare the goethite samples in this study. The reagent particles were vacuum dried at $100{ }^{\circ} \mathrm{C}$ for $24 \mathrm{~h}$ and then heated at different temperatures ranging from 200 to $800{ }^{\circ} \mathrm{C}$. The heating duration was $24 \mathrm{~h}$ for temperatures below $350{ }^{\circ} \mathrm{C}$, and only $1 \mathrm{~h}$ for higher temperatures. Powder X-ray diffraction (Miniflex II, Rigaku) with a $\mathrm{Cu} \mathrm{K}_{\alpha}$ source was used to characterize the phase composition. The morphology and crystal structure were analyzed using $200 \mathrm{kV}$ transmission electron microscopy (JEM-2010F, JEOL). The electron diffraction pattern was recorded using a film with camera length of $100 \mathrm{~cm}$. The lattice spacing $d$ was calculated by indexing the electron diffraction pattern and using the following equation:

$$
d=\frac{L \cdot \lambda}{R}
$$

Here, $L$ is camera length, $\lambda$ is the wavelength of electron $(0.02508 \AA)$ and $R$ is the measured distance of electron diffraction pattern. The in situ dehydration experiment of goethite was carried out to confirm the topotactic relationship between goethite and hematite. To transform the goethite to hematite, the electron beam was strongly focused on these particles for several seconds. The crystal structures were drawn using crystal-maker software, and the theoretical electron diffraction pattern was calculated using single-crystal software[18]. 
The interfacial energies of the twin boundaries of hematite were obtained from total energy calculations based on spin-polarized density functional theory (DFT), using generalized gradient approximation (GGA) with the Perdew-Burke-Ernzerhof (PBE) functional $[19,20]$ for the exchange-correlation energy, which is implemented in the plane-wave and projector augmented wave method of the Vienna Ab-initio Simulation Package (VASP) [21-26]. We applied a $600 \mathrm{eV}$ cutoff to limit the plane-wave basis set without compromising the computational accuracy. In this study, for comparison, we considered two different twin boundaries, which are called prismatic (100) and basal (001) twin boundaries. The supercells with prismatic (100) and basal (001) twin boundaries, which consist of 64 and 72 Fe atoms, respectively. For the first Brillouin zone sampling, a $3 \times 3 \times 5$ and $5 \times 5 \times 3$ Monkhorst-Pack special $k$-point grid with a Gaussian smearing model ( $\sigma=0.05 \mathrm{eV})$ was used for the prismatic (100) and basal (001) twin boundaries, respectively. The on-site Coulomb interaction of the strongly localized correlated d electrons of Fe was treated based on formalisms proposed by Dudarev et al.[27], with an effective on-site Coulomb interaction parameter of $4.3 \mathrm{eV}$ [28]. The calculated lattice constants of hematite were $a=5.068 \AA$ and $c=13.876 \AA$, obtained by using a $5 \times 5 \times 3 k$-mesh on the rhombohedral unit cell with 12 Fe atoms. The antiferromagnetic magnetic system was treated using the same procedures reported in [29].

The interfacial energy of the corresponding twin boundary $\gamma$ was calculated as follows:

$$
\gamma=\frac{\left(E_{\mathrm{twin}}-n E_{\mathrm{bulk}}\right)}{2 A}
$$

where $E_{\text {twin }}$ is the total energy of the system with a twin boundary, $E_{\text {bulk }}$ is the energy of a single unit of hematite in the bulk, $n$ is the number of units in the corresponding system with a twin boundary, and $A$ is the area of the interfacial plane of the twin boundary. Atomic positions were optimized until the forces on each atom were smaller than $0.02 \mathrm{eV} / \AA$ which provides with sufficient accuracy. 


\section{Results and discussions}

Figure 1a shows the XRD patterns of goethite samples that were dehydrated at various temperatures, indicating that the transformation of goethite occurred at temperatures above $230-250{ }^{\circ} \mathrm{C}$. This dehydration temperature is in agreement with previously reported results [9]. Gualtieri and Venturelli reported the formation of an intermediate phase called "protohematite" under high temperatures, by using in situ synchrotron X-ray powder diffraction [10]. In our study, this phase did not appeared because the measurement of X-ray and electron diffraction was carried out under room temperature. Owing to the crystal growth, the peaks became sharper as the temperature was increased. At temperatures between 250 and $600{ }^{\circ} \mathrm{C}$, the reflection peak of $(104)_{\mathrm{H}}$ was broader than that of $(110)_{\mathrm{H}}$. The mean crystal sizes along $(104)_{\mathrm{H}}$ and $(110)_{\mathrm{H}}$ were determined by using Scherrer's equation [30],

$$
D=\frac{K \cdot \lambda}{\beta \cdot \cos \theta}
$$

where $\mathrm{D}$ is the length of the crystal in a direction perpendicular to the reflection planes of $h, k$, and $l$; $\lambda$ is the $\mathrm{Cu} \mathrm{K}_{\alpha} \mathrm{X}$-ray wavelength (1.54184 $\AA$ ); $K$ is a constant (0.9) used in this study; $\beta$ is the peak width at half maximum; and $\theta$ is the Bragg angle of the crystal. Figure 1(b) summarizes the mean calculated crystallite size after dehydration at different temperatures. The difference in the mean crystallite size was caused by the generation of slit-shaped pores along the $[100]_{\mathrm{H}}$ direction and the preferred crystal orientation [12, 31, 32]. Figure 2 shows a TEM image of the slit-like pores generated at $250{ }^{\circ} \mathrm{C}$, with heating provided by an electric furnace. Using the contrast profile along the horizontal line shown in Figure 2(a), the slit width was estimated to be 0.8-2.5 nm, while the slit distance was determined to be approximately $4 \mathrm{~nm}$. These slit-like pores were generated from the removal of the $\mathrm{OH}$ groups in the closely arranged $[001]_{\mathrm{G}}$ direction.

The in situ dehydration experiment of goethite was carried out to confirm the 
topotactic relationship between goethite and hematite. Figure 3(a) shows TEM image and HR-TEM image of a goethite rod-like crystal before dehydration and the corresponding electron diffraction pattern, which clearly indicates that the rod-like crystal was a single crystal in the growth direction of $[001]_{G}$. To transform the goethite to hematite, the electron beam was strongly focused on this particle for several seconds; Figure 3(b) shows a TEM image of the sample in Figure 3(a) after dehydration. During dehydration, slit-like pores were generated as shown in Fig.2 because the $\mathrm{OH}$ groups with closely arranged $[001]_{\mathrm{G}}$ axes were removed, as shown in the structural model in Figure 6(a). None of the HR-TEM images or electron diffraction patterns correspond to single-crystalline hematite, in which the many twin boundaries were transformed from goethite after dehydration. Although this twin structure was already investigated by Watari et al. [17], we reconfirmed the structural relationship using both crystal-maker software and crystal-diffraction calculations.

Figure 4 shows the crystal structure of hematite projected through the [010] direction, where the calculated electron diffraction patterns are shown in the bottom row. When hematite was projected through the [010] direction, two non-equivalent atomic arrangements were available, referred to as the (a) observed structure and (b) reversed structure. When these two arrangements were introduced into the twinned crystallographic structure, the atomic arrangement and resulting diffraction pattern are illustrated in Figure 4(c). The d spacings were calculated from the electron diffraction patterns and fast Fourier transformation (FFT) of HR-TEM image, as shown in Figure 5. The calculated d spacings are summarized in Table 1. Although the experimental data contained measurement error, the observed structure shown in Figure 3(b) agreed well with the calculated result from the structure model shown in Figure 4(c). Thus, it is clear that such twins were formed in hematite.

Based on the electron diffraction patterns shown in Fig. 3, the crystallographic 
orientation relationship between goethite and hematite was investigated. Figure 6 shows the atomic structure corresponding to the orientation relationship represented by $(040)_{\mathrm{G}}$ // $(006)_{\mathrm{H}}$ and $(002)_{\mathrm{G}} / /(300)_{\mathrm{H}}$. Table 2 shows the lattice spacing of goethite and hematite corresponding to Figure 6. Owing to the removal of $\mathrm{OH}$ groups, the distance of the (006) $)_{\mathrm{H}}$ plane was decreased.

We confirmed the detailed crystal structure of hematite including twin boundary after dehydration by electron beam irradiation as well as thermal dehydration in an electric furnace. Figure 7(a) shows a high-resolution TEM (HR-TEM) image corresponding to the electron diffraction and FFT patterns of the sample that was dehydrated at $250{ }^{\circ} \mathrm{C}$ for a period of over $24 \mathrm{~h}$. The TEM image indicates that the crystal size ranged from 10 to $30 \mathrm{~nm}$, which agrees with the crystal size obtained by XRD shown in Fig. 1(b). The [010] projected electron diffraction pattern recorded over the entire area of particles shows a twin structure similar to that shown in Figure 3(b), suggesting formation of a twin structure. In the TEM image shown in Figure 7(a), we have chosen the region on the right hand side of the image because it has no overlapping grains. We carefully observed the lattice fringe and distinguished the grain boundaries shown by the solid black line. The FFT patterns obtained from each grain demonstrated that the goethite particle consisted of small single crystalline grains extending toward $[100]_{\mathrm{H}}$, where the observed and reversed structures (see Figure 4) appeared alternately, as shown in Figure 7(b), in which red and blue correspond to the observed and reversed structure of hematite, respectively. According to the enlarged HR-TEM image in Figure 7(c), the twin boundary consisted of a straight line on the $(100)_{\mathrm{H}}$ plane, in which the Fe atomic positions were well matched in each twin crystal (Figure $7(d)$ ).

HR-TEM observations were carried out for the sample that was dehydrated at $800{ }^{\circ} \mathrm{C}$ for $1 \mathrm{~h}$, as shown in Figure 8 . Compared to the case of $250{ }^{\circ} \mathrm{C}$, crystal size 
increased due to crystal growth at higher temperature. Under these dehydration conditions, most of the twins disappeared; however, some hematite particles remained in the twin structure. Shown in Figure 8(a), the rod-like particle consisted of several grains with size of over $100 \mathrm{~nm}$. These grains were separated from the (100) related planes and alternately appeared, just like the twins that resulted from dehydration at $250^{\circ} \mathrm{C}$. In addition, we investigated the contrast near the grain boundary and discovered two types of grain boundaries, edge-on and inclined grain boundaries, as shown in Figure 9(a, b). In the crystal structural model of hematite in the [001] projection shown in Figure 9(c), three dashed lines corresponding to the crystallographically equivalent (010), (100) and (11̄0) planes represent the possible grain boundaries. Therefore, the twin boundary could exist not only as a single plane, but also as a facet structure, as shown in Figure 9(d).

In general, interfaces like grain and twin boundaries tend to disappear during crystal grain growth at high temperature to decrease the interfacial energies. Interfacial energies depend on the boundary characteristics, which are determined by the crystallographic orientation relationship between two crystal grains. Compared to random boundaries, twin boundaries are known to have lower interfacial energy owing to their well-matched atomic structures. To investigate why the (100) twin boundary was present at high temperature in our samples, we calculated the interfacial energies of prismatic (100) screw and basal (001) rotation interfaces. Our results showed that the twin boundary of prismatic (100) screw had lower interfacial energy $\left(0.24 \mathrm{~J} / \mathrm{m}^{2}\right)$ than that of the basal (001) rotation interface $\left(1.31 \mathrm{~J} / \mathrm{m}^{2}\right)$; these values agree with the interfacial energies of other similar corundum structures like $\alpha \mathrm{Al}_{2} \mathrm{O}_{3}$ and $\alpha \mathrm{Cr}_{2} \mathrm{O}_{3}$ [33-35]. In the case of $\alpha \mathrm{Cr}_{2} \mathrm{O}_{3}$, the grain boundary of prismatic (100) screw had the smallest interfacial energy of $0.24 \mathrm{~J} / \mathrm{m}^{2}$ among other grain-boundary symmetries of prismatic (100) glide, basal (001) rotation, and others. Owing to this lower interfacial energy, the prismatic (100) screw interface remained after higher-temperature treatment at $800{ }^{\circ} \mathrm{C}$.

Finally, we propose a mechanism, shown in Figure 9(e), for the transformation of 
goethite into hematite containing twin boundaries with a slit-like porous morphology during thermal decomposition. At the initial stage of goethite dehydration, many hematite grains with small size nucleated and the crystal structure of the hematite nucleus was randomly distributed between the observed and reversed structures owing to their equivalence. After dehydration was complete, the twin boundaries were formed at the interface between the observed and reversed phases. By increasing the temperature, sintering for each particle grain occurred, resulting in both crystal growth and the disappearance of pores. However, the twin structure was partially present at $800^{\circ} \mathrm{C}$.

\section{Conclusion}

We investigated twin formation in hematite during the dehydration of goethite. After electron-beam irradiation or thermal decomposition at $250{ }^{\circ} \mathrm{C}$, slit-like pores that included twin boundaries were formed in hematite. Crystal growth occurred and the pores nearly disappeared as the temperature was increased, as reported in previous studies. TEM observation clarified that the single-crystalline goethite, with the growth direction of $[001]_{\mathrm{G}}$, demonstrated transformation into twin-boundary-induced hematite with the corresponding direction of $[100]_{\mathrm{H}}$, where the twin boundaries were crystallographically equivalent (100), (010), and (1 $\overline{1} 0)$ planes. By using DFT calculation, the twin boundary of prismatic (100) screw was shown to have a low interfacial energy $\left(0.24 \mathrm{~J} / \mathrm{m}^{2}\right)$. During dehydration, many hematite grains with small size were generated, where the crystal structure of the hematite nucleus was randomly selected from the observed and reversed structures owing to their equivalence. Because of its low interfacial energy, the prismatic (100) screw interface remained as the twin boundary after higher-temperature treatment at $800{ }^{\circ} \mathrm{C}$. 


\section{Acknowledgement}

The TEM observations were conducted at Hokkaido University and supported by the “Nanotechnology Platform” Program of the Ministry of Education, Culture, Sports, Science and Technology of Japan (MEXT).

\section{References}

[1] U. Schwertmann, R.M. Cornell, Iron Oxides in the Laboratory, Wiley2000.

[2] R.M. Cornell, U. Schwertmann, The Iron Oxides, The Iron Oxides, Wiley-VCH Verlag GmbH \& Co. KGaA2004, pp. i-xx.

[3] G. Busca, M. Daturi, E. Finocchio, V. Lorenzelli, G. Ramis, R.J. Willey, Transition metal mixed oxides as combustion catalysts: preparation, characterization and activity mechanisms, Catal. Today, 33 (1997) 239-249.

[4] J.M.D. Coey, Magnetic Properties of Iron in Soil Iron Oxides and Clay Minerals, in: J.W. Stucki, B.A. Goodman, U. Schwertmann (Eds.) Iron in Soils and Clay Minerals, Springer Netherlands1988, pp. 397-466.

[5] R.B. Cahyono, G. Saito, N. Yasuda, T. Nomura, T. Akiyama, Porous Ore Structure and Deposited Carbon Type during Integrated Pyrolysis-Tar Decomposition, Energy \& Fuels, 28 (2014) 2129-2134.

[6] C. Goss, The kinetics and reaction mechanism of the goethite to hematite transformation, Mineralogical Magazine, 51 (1987) $437-451$.

[7] H.D. Ruan, R.L. Frost, J.T. Kloprogge, L. Duong, Infrared spectroscopy of goethite dehydroxylation. II. Effect of aluminium substitution on the behaviour of hydroxyl units, Spectrochimica Acta Part A: Molecular and Biomolecular Spectroscopy, 58 (2002) 479-491.

[8] G.N. Kustova, E.B. Burgina, V.A. Sadykov, S.G. Poryvaev, Vibrational spectroscopic investigation of the goethite thermal decomposition products, Phys. Chem. Miner., 18 379-382.

[9] H. Liu, T. Chen, X. Zou, C. Qing, R.L. Frost, Thermal treatment of natural goethite: Thermal transformation and physical properties, Thermochim. Acta, 568 (2013) 115-121.

[10] A.F. Gualtieri, P. Venturelli, In situ study of the goethite-hematite phase transformation by real time synchrotron powder diffraction, Am. Mineral., 84 (1999) 895-904.

[11] F. Watari, P. Delavignette, J. Van Landuty, S. Amelinckx, Electron microscopic study of dehydration transformations. Part III: High resolution observation of the reaction process FeOOH $\rightarrow$ Fe2O3, J. Solid State Chem., 48 (1983) 49-64. 
[12] H. Naono, R. Fujiwara, Micropore formation due to thermal decomposition of acicular microcrystals of $\alpha$-FeOOH, J. Colloid Interface Sci., 73 (1980) 406-415

[13] J. Lima-de-Faria, Dehydration of goethite and diaspore, Zeitschrift für Kristallographie-Crystalline Materials, 119 (1964) $176-203$.

[14] H. Yang, R. Lu, R.T. Downs, G. Costin, Goethite, [alpha]-FeO(OH), from single-crystal data, Acta Crystallographica Section E, 62 (2006) i250-i252.

[15] G. Oosterhout, Morphology of synthetic sub-microscopic crystals of [alpha] and [gamma]FeOOH and of [gamma]Fe2O3 prepared from FeOOH, Acta Crystallogr., 13 (1960) 932-935.

[16] M. Francombe, H. Rooksby, Structure transformations effected by the dehydration of diaspore, goethite and delta ferric oxide, Clay Min. Bull, 4 (1959) $1-14$.

[17] F. Watari, J. Van Landuyt, P. Delavignette, S. Amelinckx, Electron microscopic study of dehydration transformations. I. Twin formation and mosaic structure in hematite derived from goethite, J. Solid State Chem., 29 (1979) 137-150.

[18] D. Palmer, CrystalMaker CrystalMaker Software Ltd, Yarnton, Oxfordshire, England, DOI (2009).

[19] J.P. Perdew, K. Burke, M. Ernzerhof, Generalized Gradient Approximation Made Simple, Phys. Rev. Lett., 77 (1996) $3865-3868$.

[20] J.P. Perdew, K. Burke, M. Ernzerhof, Generalized Gradient Approximation Made Simple [Phys. Rev. Lett. 77, 3865 (1996)], Phys. Rev. Lett., 78 (1997) 1396-1396.

[21] G. Kresse, J. Hafner, ¥textit\{Ab initio\} molecular dynamics for liquid metals, Physical Review B, 47 (1993) 558-561.

[22] G. Kresse, J. Hafner, ¥textit\{Ab initio\} molecular-dynamics simulation of the liquid-metal¥char21\{\}amorphous-semiconductor transition in germanium, Physical Review B, 49 (1994) 14251-14269.

[23] G. Kresse, J. Furthmüller, Efficiency of ab-initio total energy calculations for metals and semiconductors using a plane-wave basis set, Computational Materials Science, 6 (1996) 15-50.

[24] P.E. Blöchl, Projector augmented-wave method, Physical Review B, 50 (1994) 17953-17979.

[25] G. Kresse, J. Furthmüller, Efficient iterative schemes for ¥textit\{ab initio\} total-energy calculations using a plane-wave basis set, Physical Review B, 54 (1996) 11169-11186.

[26] G. Kresse, D. Joubert, From ultrasoft pseudopotentials to the projector augmented-wave method, Physical Review B, 59 (1999) $1758-1775$.

[27] S.L. Dudarev, G.A. Botton, S.Y. Savrasov, C.J. Humphreys, A.P. Sutton, Electron-energy-loss spectra and the structural stability of nickel oxide: An LSDA+U study, Physical Review B, 57 (1998) 1505-1509.

[28] N.J. Mosey, P. Liao, E.A. Carter, Rotationally invariant ab initio evaluation of Coulomb and exchange parameters for DFT+U calculations, The Journal of Chemical Physics, 129 (2008) 014103 
[29] G. Rollmann, A. Rohrbach, P. Entel, J. Hafner, First-principles calculation of the structure and magnetic phases of hematite, Physical Review B, 69 (2004) 165107.

[30] P. Scherrer, Bestimmung der Grösse und der inneren Struktur von Kolloidteilchen mittels Röntgenstrahlen, Nachrichten von der Gesellschaft der Wissenschaften zu Göttingen, mathematisch-physikalische Klasse, 1918 (1918) 98-100.

[31] S. Gialanella, F. Girardi, G. Ischia, I. Lonardelli, M. Mattarelli, M. Montagna, On the goethite to hematite phase transformation, J. Therm. Anal. Calorim., 102 (2010) 867-873.

[32] F. Jia, K. Ramirez-Muñiz, S. Song, Mechanism of the formation of micropores in the thermal decomposition of goethite to hematite, Surf. Interface Anal., 47 (2015) 535-539.

[33] A.G. Marinopoulos, C. Elsässer, Microscopic structure and bonding at the rhombohedral twin interface in $\alpha$-Al2O3, Acta Mater., 48 (2000) 4375-4386.

[34] A.G. Marinopoulos, S. Nufer, C. Elsässer, Interfacial structures and energetics of basal twins in \$类suremath $\{$ alpha $\}-\{¥ m a t h r m\{\mathrm{Al}\}\}_{-}\{2\}\{¥ \operatorname{mathrm}\{\mathrm{O}\}\}_{-}\{3\}: \$$ First-principles density-functional and empirical calculations, Physical Review B, 63 (2001) 165112.

[35] S. Fabris, S. Nufer, C. Elsässer, T. Gemming, Prismatic \$¥ensuremath\{¥Sigma\}3(101¥ifmmode¥bar¥else¥textasciimacron¥fi \{\} 0$) \$$ twin boundary in $\$ ¥$ ensuremath $\{¥ a l p h a\}$ - $\{¥ \text { mathrm }\{\mathrm{Al}\}\}_{-}\{2\}\{¥ m a t h r m\{\mathrm{O}\}\}_{-}\{3\} \$$ investigated by density functional theory and transmission electron microscopy, Physical Review B, 66 (2002) 155415. 
Tables

Table 1. Lattice spacing calculated from structure model, electron diffraction and FFT shown in Fig.4.

\begin{tabular}{cccc}
\hline & (a) Structure model & (b) Electron diffraction & (c) FFT \\
\hline a : $(100)_{\mathrm{G}}$ & $4.36 \AA$ & $4.58 \AA$ & $4.60 \AA$ \\
$\mathrm{b}:(102)_{\mathrm{G}}$ & $3.69 \AA$ & $3.89 \AA$ & $3.90 \AA$ \\
$\mathrm{c}:(002)_{\mathrm{G}}$ & $6.89 \AA$ & $7.22 \AA$ & $7.15 \AA$ \\
$\theta$ & $90^{\circ}$ & $90^{\circ}$ & $90^{\circ}$ \\
\hline
\end{tabular}

Table 2. Lattice spacing of the goethite and hematite structures shown in Figure 4.

\begin{tabular}{ccc}
\hline Goethite, $G$ & Hematite, $H$ & $H / G$ \\
\hline$(040)_{G}: 2.489 \mathrm{~nm}$ & $(006)_{H}: 2.295 \mathrm{~nm}$ & 0.922 \\
$(002)_{G}: 1.512 \mathrm{~nm}$ & $(300)_{H}: 1.454 \mathrm{~nm}$ & 0.961 \\
\hline
\end{tabular}




\section{Figure captions}

Fig. 1 (a) XRD patterns of the samples after dehydration at different temperatures and durations. At $250{ }^{\circ} \mathrm{C}$, the goethite transformed to hematite. (b) Crystallite size of samples evaluated using the Scherrer equation.

Fig. 2 (a) TEM image of slit-like pores generated at $250{ }^{\circ} \mathrm{C}$. (b) Contrast profile of the horizontal line in (a). The slit width was estimated to be $0.8-2.5 \mathrm{~nm}$, while the slit distance was determined to be approximately $4 \mathrm{~nm}$.

Fig. 3 a rod-like goethite crystal was analyzed using TEM. The in situ dehydration experiment was performed by irradiating the crystal with a strongly focused electron beam. (a) TEM image, HR-TEM image and electron diffraction patterns of goethite in the growth direction of $[001]_{\mathrm{G}}$. (b) TEM image and HR-TEM image corresponding to the same region in (a) after dehydration.

Fig. 4 Crystal structure of hematite projected through the [010] direction in the (a) observed, (b) reversed, and (c) overlapping observed and reversed structures. The calculated electron diffraction patterns corresponding to (a), (b), and (c) are shown in the bottom row.

Fig. 5 Comparison of (a) calculated electron diffraction pattern, (b) measured electron diffraction pattern and (c) FFT of HR-TEM image. The calculated d spacing is shown in Table 1.

Fig. 6 Crystal structure corresponding to the orientation relationship between (a) $\alpha \mathrm{FeOOH}$ (goethite) and (b) $\alpha \mathrm{Fe}_{2} \mathrm{O}_{3}$ (hematite). The calculated electron diffraction patterns corresponding to (a) and (b) are shown in the bottom.

Fig. 7 (a) HR-TEM images of the sample dehydrated at $250{ }^{\circ} \mathrm{C}$ for $24 \mathrm{~h}$. The [010] projected electron diffraction pattern measured over the entire area of particles shows formation of a twin structure similar to that shown in Figure 3. The particles consisting of small, rod-like single crystals are marked by a solid black line, and the FFT pattern analyzed at each single crystal is shown on the right. (b) Schematic illustration of the structure in (a), in which red and blue correspond to the observed and reversed structure of hematite, respectively. (c) Enlarged HR-TEM image and (d) schematic of the twin boundary.

Fig. 8 (a) TEM image of the sample that was dehydrated at $800{ }^{\circ} \mathrm{C}$ for $1 \mathrm{~h}$. The [010] projected electron diffraction pattern measured from the entire area of particles shows a twin structure similar to that shown in Figure 6. The particle displayed a zebra-striped contrast corresponding to the single crystal. The FFT pattern of each area is shown on the right. (b) Schematic illustration of the structure in (a), in which red and blue correspond to the observed and reversed structure of hematite, respectively. 
Fig. 9 (a, b) TEM images of the twin boundary of the sample that was dehydrated at $800{ }^{\circ} \mathrm{C}$ for $1 \mathrm{~h}$, showing that both edge-on and inclined twin boundaries were present. (c) Crystal structure of hematite in the [001] projection, where the dashed lines show the possible planes of the twin boundary. (d) Schematic illustration of the two possible twin boundaries. (e) Mechanism for twin-induced hematite formation through the dehydration of goethite. 

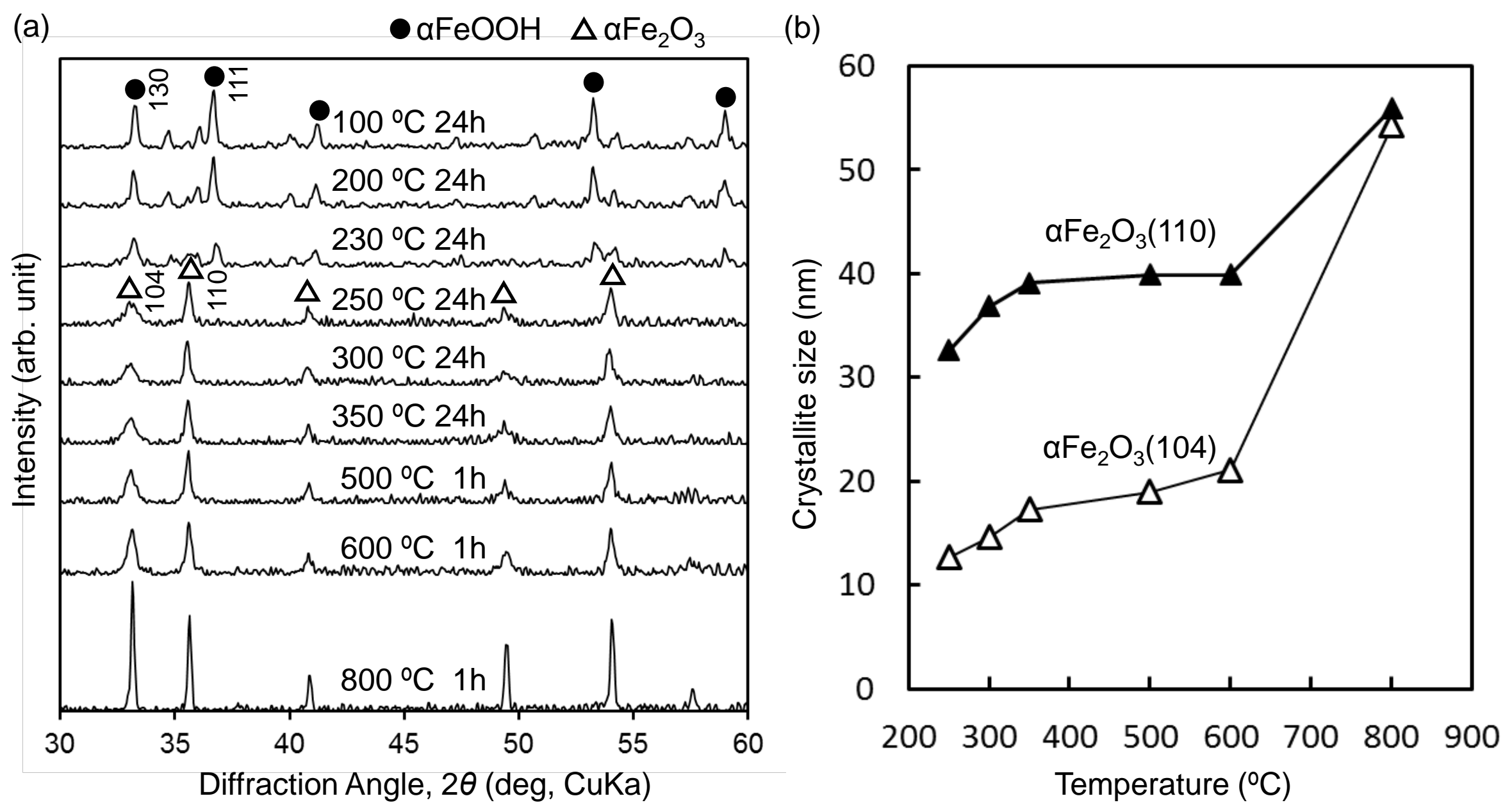

Fig. 1 (a) XRD patterns of the samples after dehydration at different temperatures and durations. At $250^{\circ} \mathrm{C}$, the goethite transformed to hematite. (b) Crystallite size of samples evaluated using the Scherrer equation. 

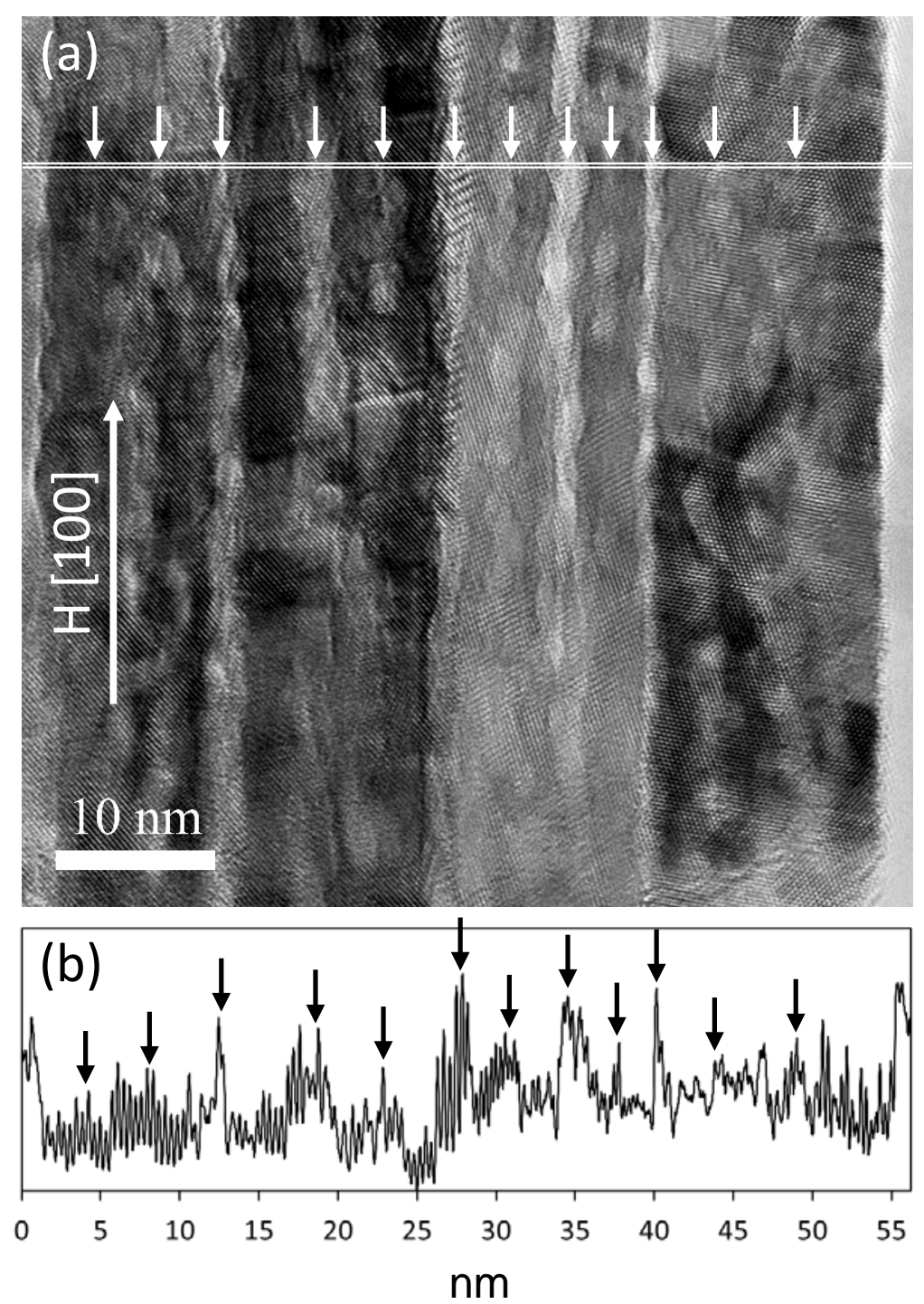

Fig. 2 (a) TEM image of slit-like pores generated at $250^{\circ}$ C. (b) Contrast profile of the horizontal line in (a). The slit width was estimated to be $0.8-2.5 \mathrm{~nm}$, while the slit distance was determined to be approximately $4 \mathrm{~nm}$. 
(a) Before dehydration ( $\alpha \mathrm{FeOOH})$

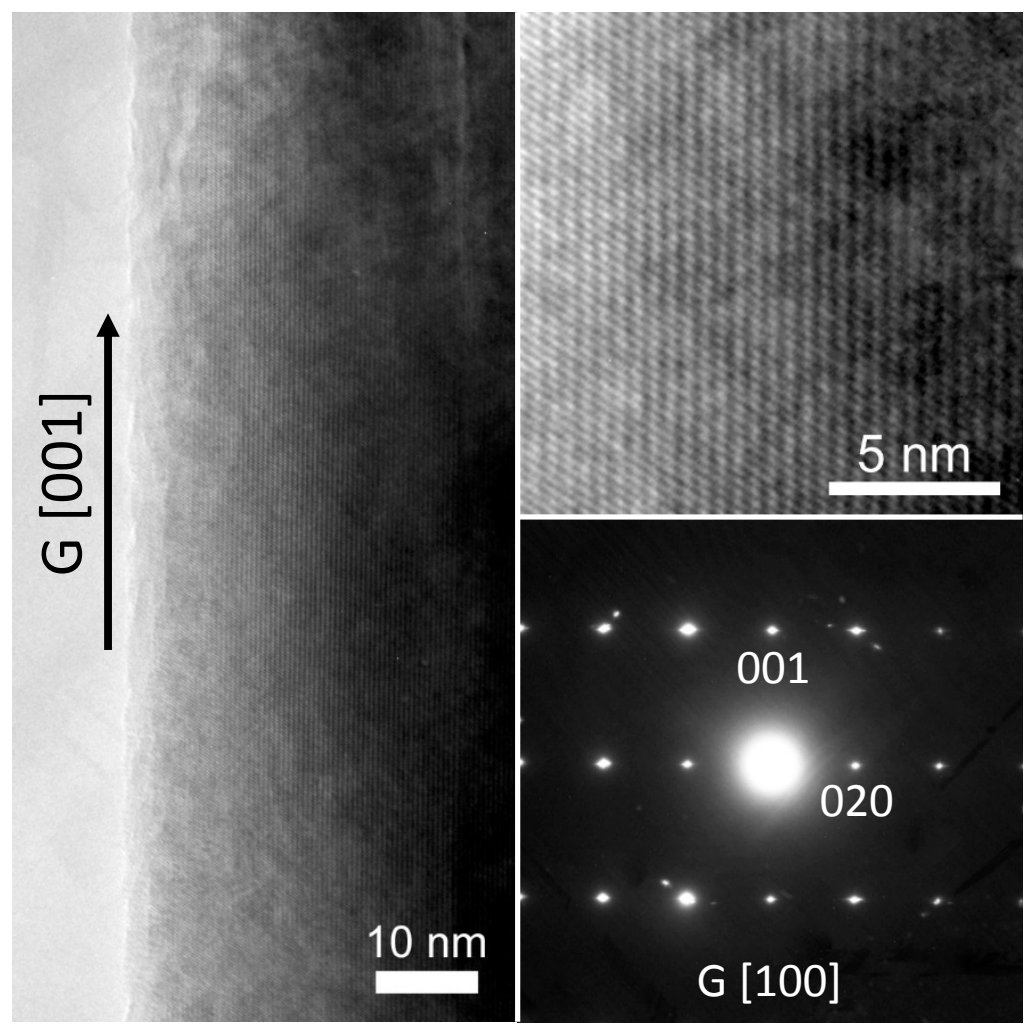

(b) After dehydration $\left(\alpha \mathrm{Fe}_{2} \mathrm{O}_{3}\right)$

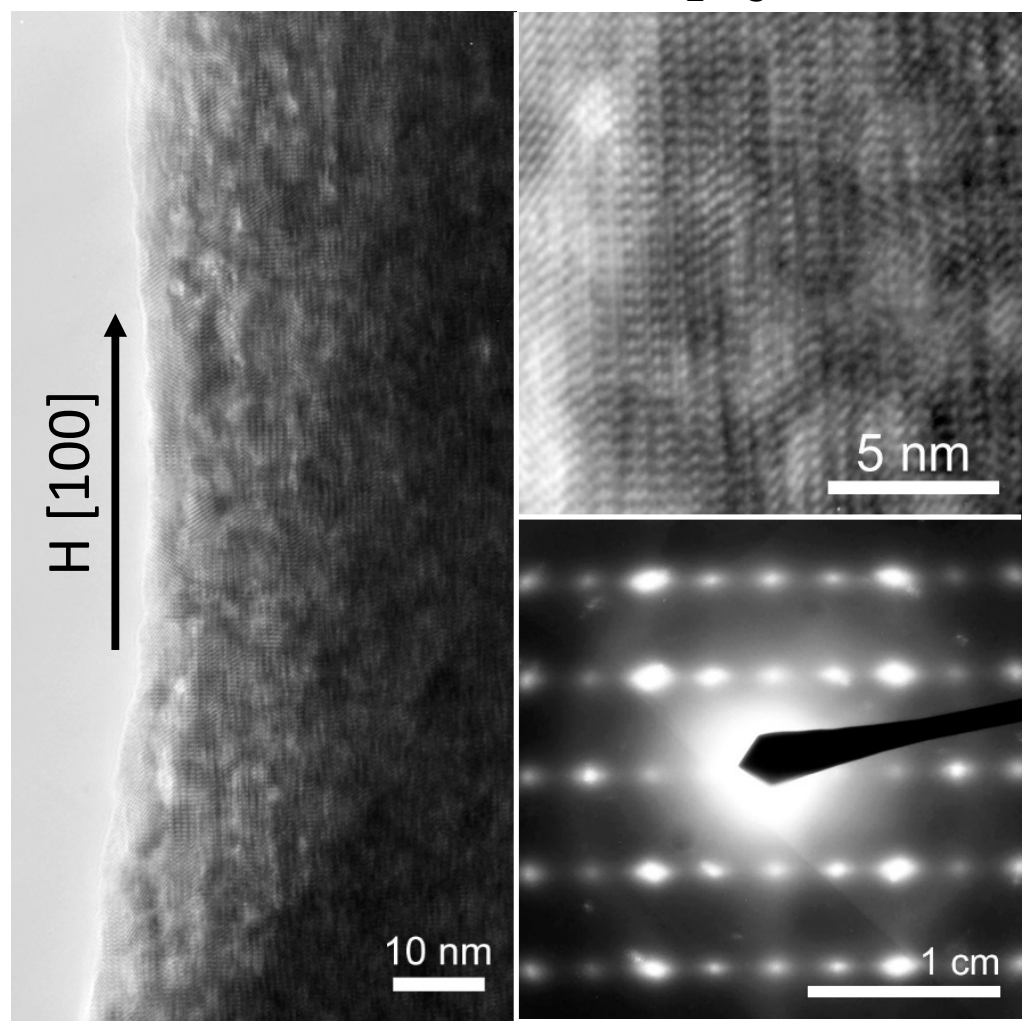

Fig. 3 a rod-like goethite crystal was analyzed using TEM. The in situ dehydration experiment was performed by irradiating the crystal with a strongly focused electron beam. (a) TEM image, HR-TEM image and electron diffraction patterns of goethite in the growth direction of $[001]_{G}$. (b) TEM image and HR-TEM image corresponding to the same region in (a) after dehydration. 
(a) Observed $\alpha \mathrm{Fe}_{2} \mathrm{O}_{3} \quad \mathrm{Fe} \mathrm{O}$

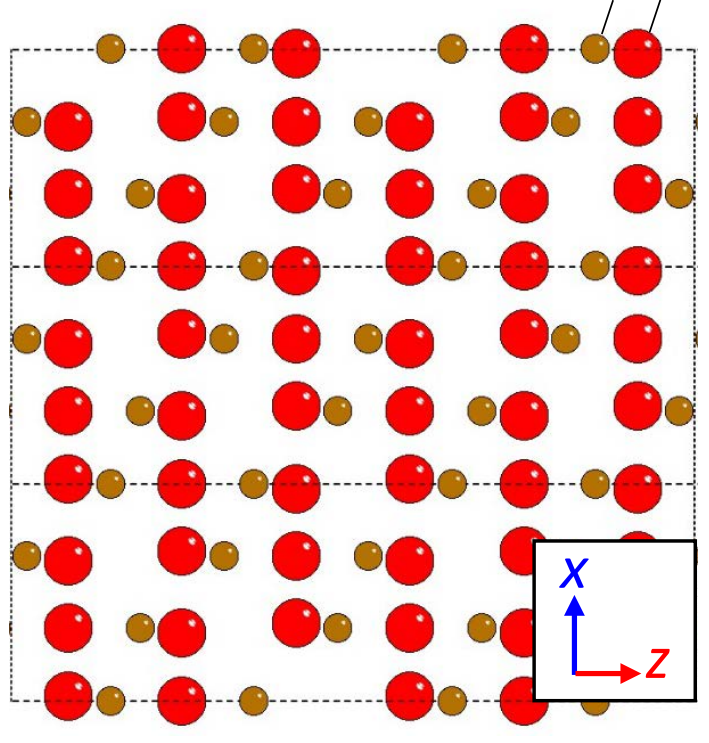

$\bullet$

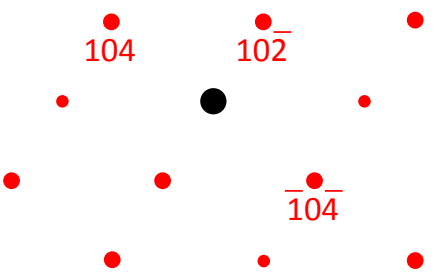

(b) Reversed $\alpha \mathrm{Fe}_{2} \mathrm{O}_{3}$
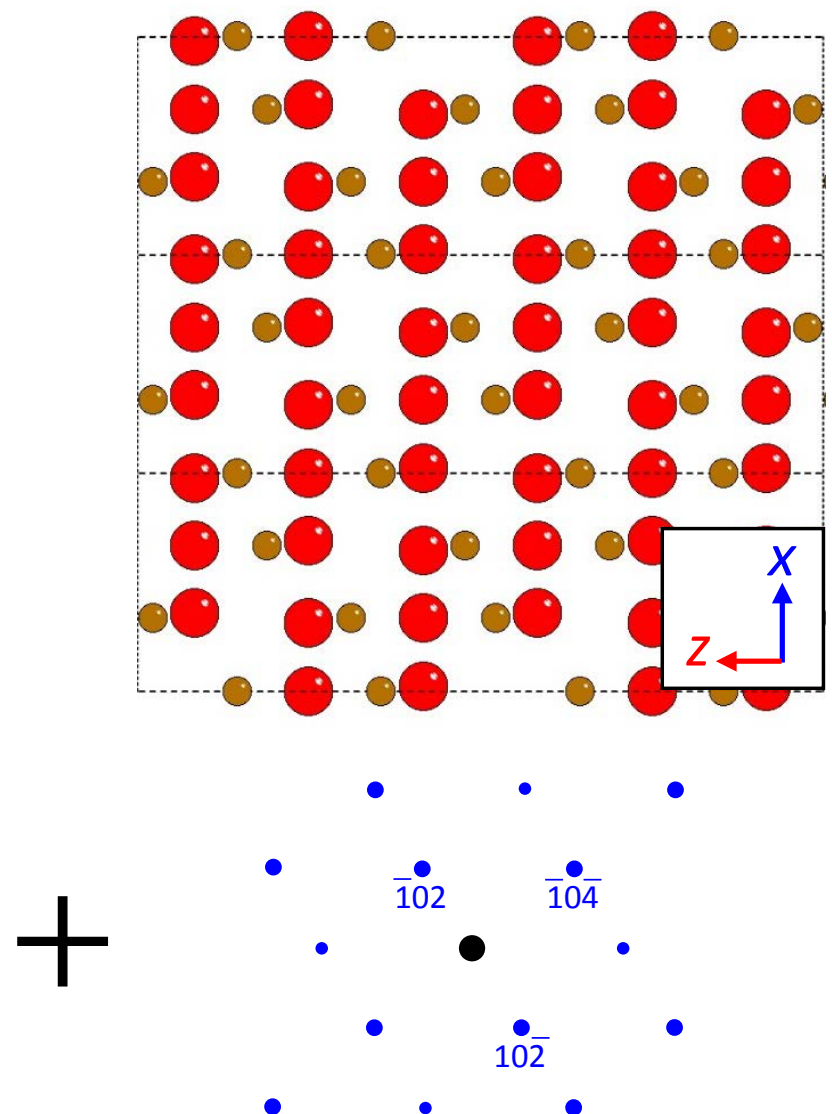

(c) Observed + Reversed $\odot \circ \odot 00.00$ 00000000000 0000000000000 - -0 - $\odot \circ$ 000000000000 00.000000000 $-00-000$ 00000000000 00.000000000 - -000000

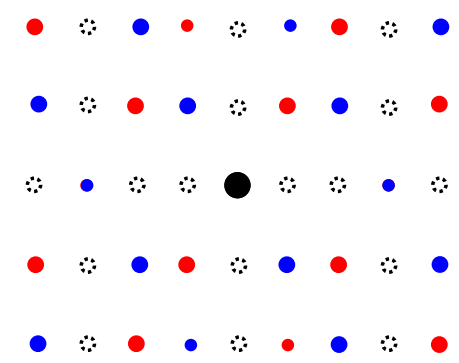

Fig. 4 Crystal structure of hematite projected through the [010] direction in the (a) observed, (b) reversed, and (c) overlapping observed and reversed structures. The calculated electron diffraction patterns corresponding to (a), (b), and (c) are shown in the bottom row. 


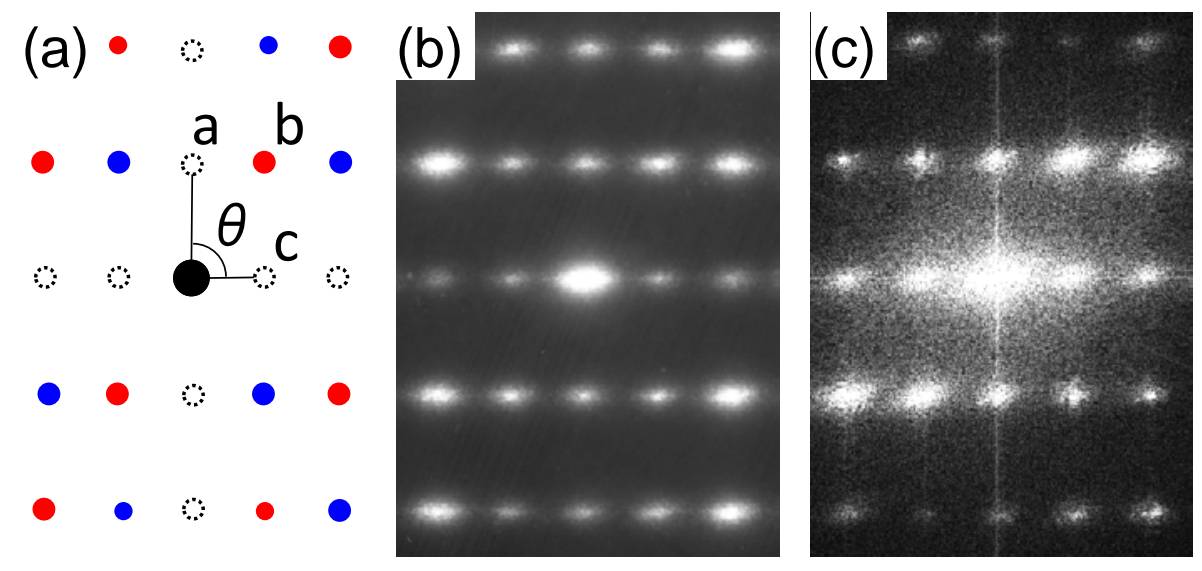

Fig. 5 Comparison of (a) calculated electron diffraction pattern, (b) measured electron diffraction pattern and (c) FFT of HR-TEM image. The calculated d spacing is shown in Table 1. 


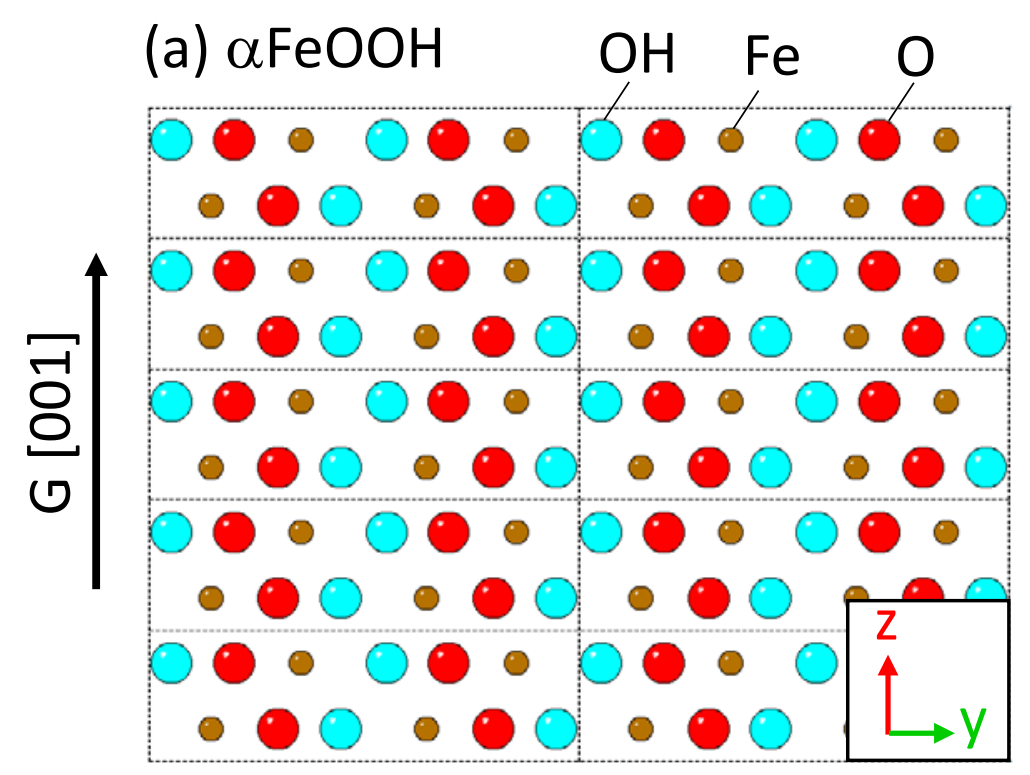

(b) $\alpha \mathrm{Fe}_{2} \mathrm{O}_{3}$
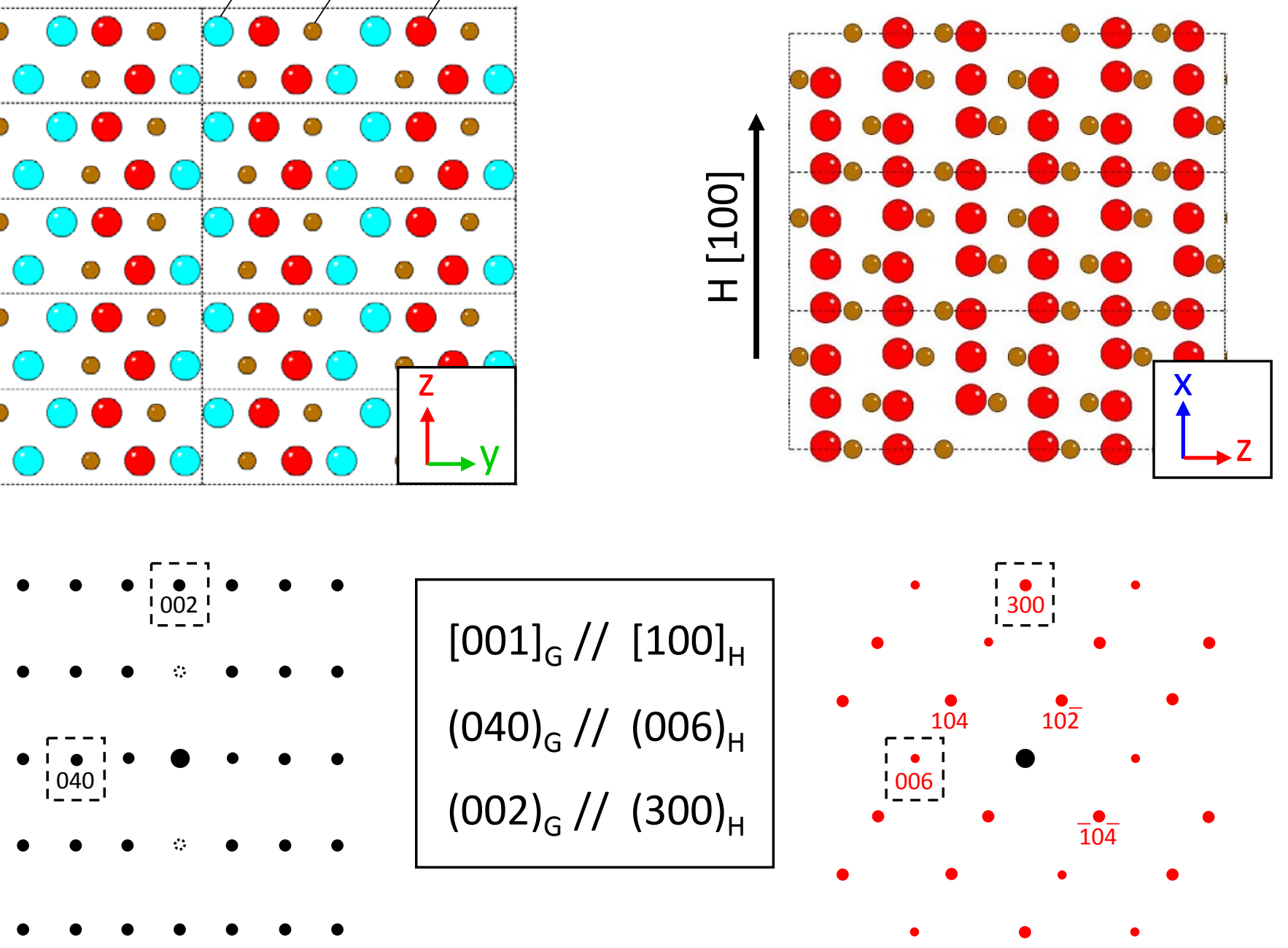

Fig. 6 Crystal structure corresponding to the orientation relationship between (a) $\alpha \mathrm{FeOOH}$ (goethite) and (b) $\alpha \mathrm{Fe}_{2} \mathrm{O}_{3}$ (hematite). The calculated electron diffraction patterns corresponding to (a) and (b) are shown in the bottom. 

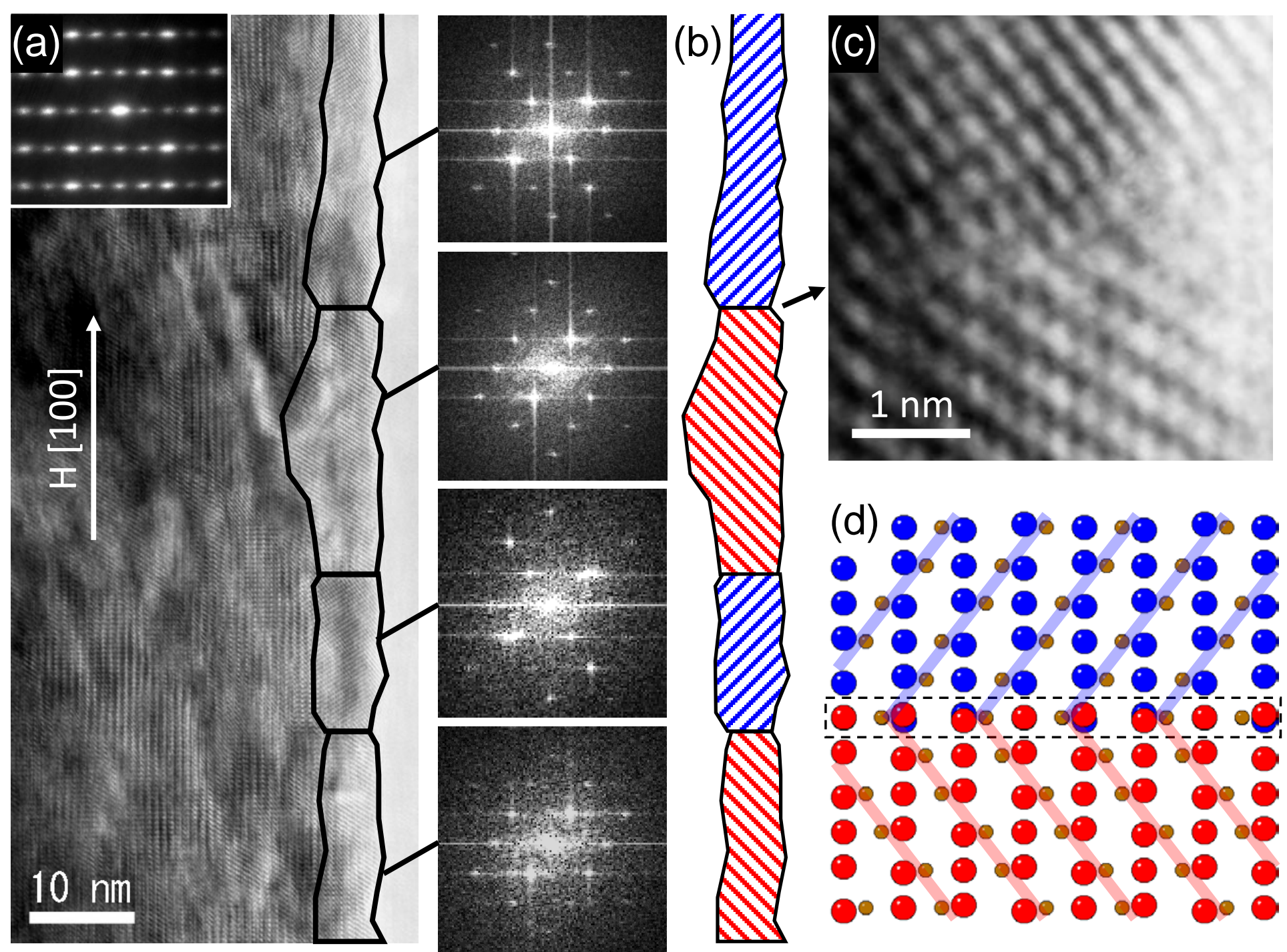

Fig. 7 (a) HR-TEM images of the sample dehydrated at $250^{\circ} \mathrm{C}$ for $24 \mathrm{~h}$. The [010] projected electron diffraction pattern measured over the entire area of particles shows formation of a twin structure similar to that shown in Figure 3. The particles consisting of small, rod-like single crystals are marked by a solid black line, and the FFT pattern analyzed at each single crystal is shown on the right. (b) Schematic illustration of the structure in (a), in which red and blue correspond to the observed and reversed structure of hematite, respectively. (c) Enlarged HR-TEM image and (d) schematic of the twin boundary. 


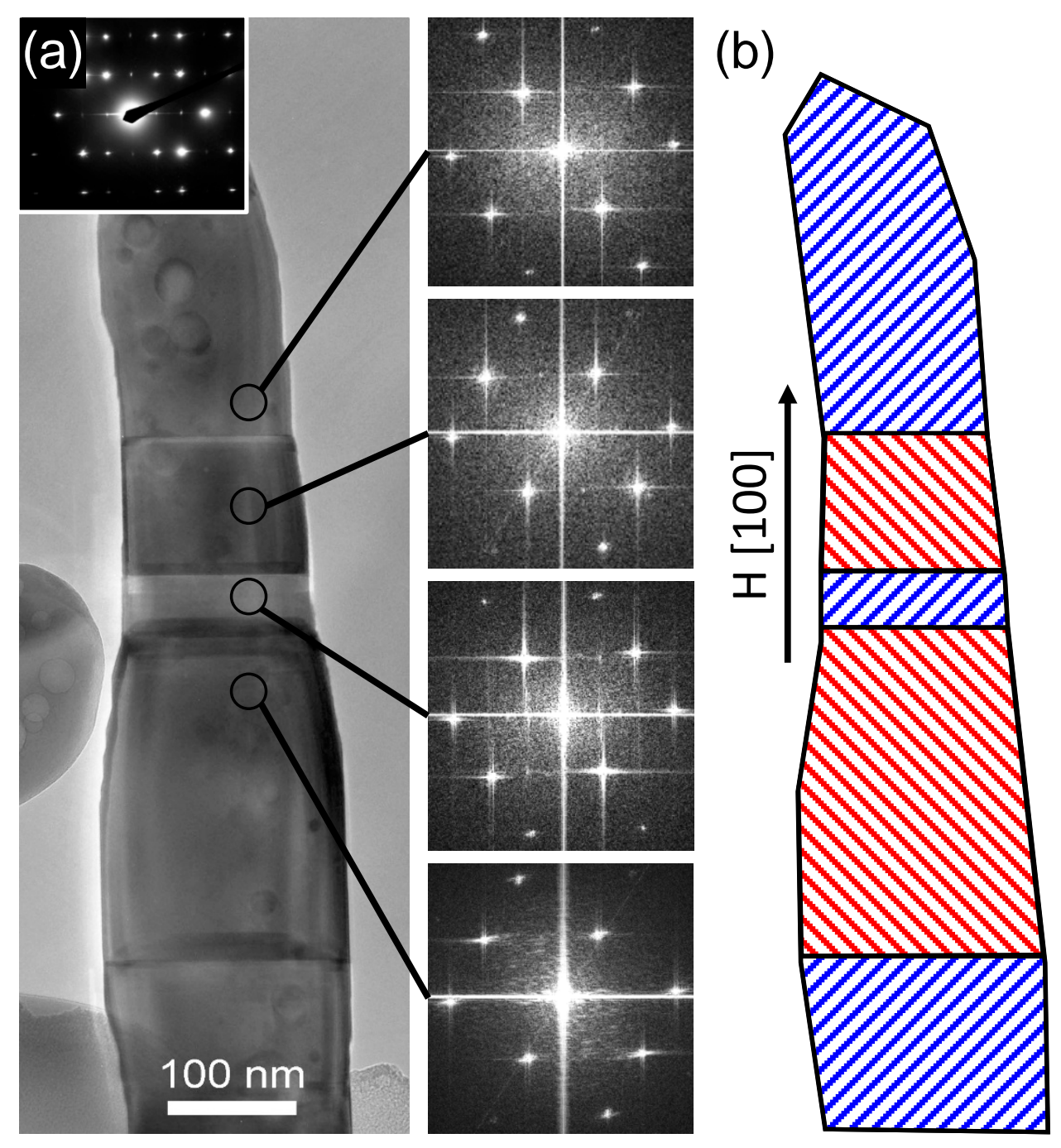

Fig. 8 (a) TEM image of the sample that was dehydrated at $800^{\circ} \mathrm{C}$ for $1 \mathrm{~h}$. The [010] projected electron diffraction pattern measured from the entire area of particles shows a twin structure similar to that shown in Figure 6 . The particle displayed a zebra-striped contrast corresponding to the single crystal. The FFT pattern of each area is shown on the right. (b) Schematic illustration of the structure in (a), in which red and blue correspond to the observed and reversed structure of hematite, respectively. 
(a) Edge-on
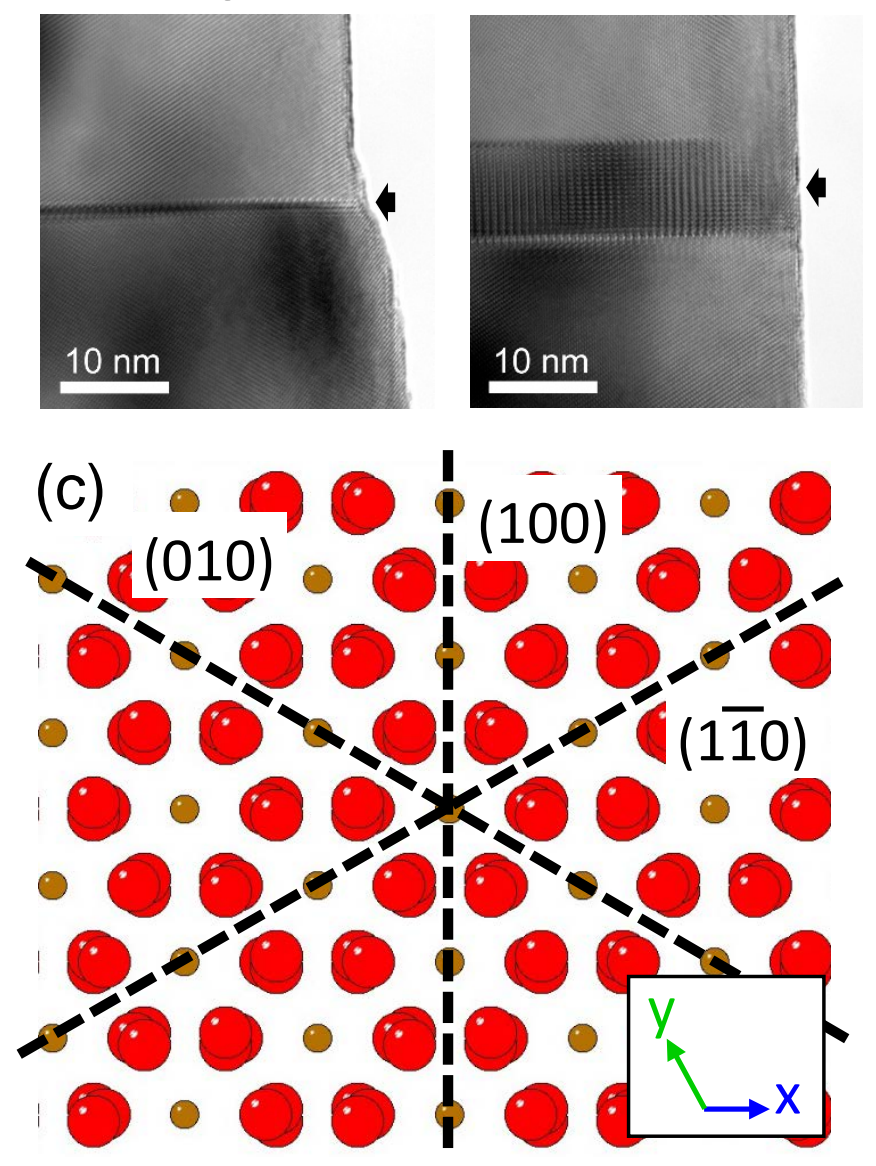

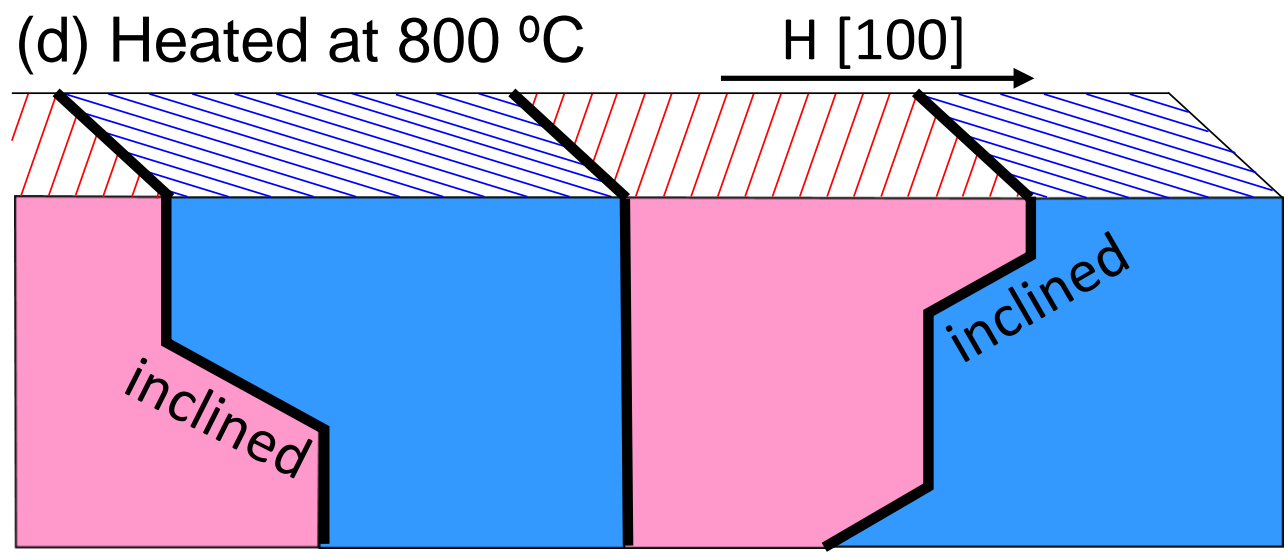

Edge-on

(e)

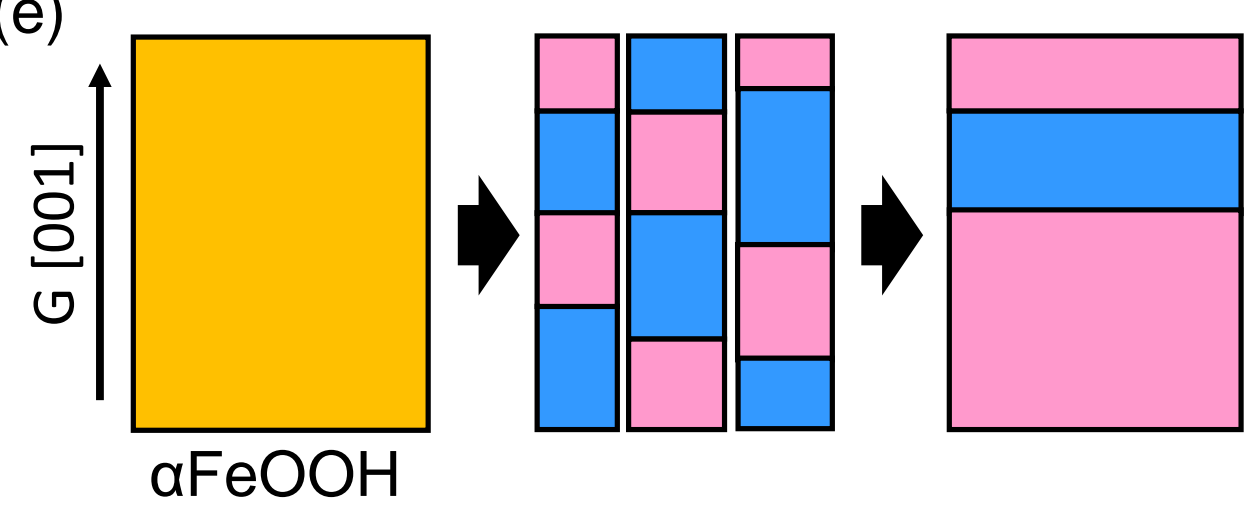

Fig. 9 (a, b) TEM images of the twin boundary of the sample that was dehydrated at $800^{\circ} \mathrm{C}$ for $1 \mathrm{~h}$, showing that both edge-on and inclined twin boundaries were present. (c) Crystal structure of hematite in the [001] projection, where the dashed lines show the possible planes of the twin boundary. (d) Schematic illustration of the two possible twin boundaries. (e) Mechanism for twin-induced hematite formation through the dehydration of goethite. 